\title{
Genetic Variants and Systemic Complement Activation Levels Are Associated With Serum Lipoprotein Levels in Age-Related Macular Degeneration
}

\author{
Constantin C. Paun, ${ }^{1,2}$ Lebriz Ersoy, ${ }^{3}$ Tina Schick, ${ }^{3}$ Joannes M. M. Groenewoud, ${ }^{4}$ \\ Yara T. Lechanteur, ${ }^{1}$ Sascha Fauser, ${ }^{3}$ Carel B. Hoyng, ${ }^{1}$ Eiko K. de Jong, ${ }^{1,2}$ \\ and Anneke I. den Hollander ${ }^{1,2,5}$ \\ ${ }^{1}$ Department of Ophthalmology, Radboud University Medical Center, Nijmegen, The Netherlands
2Radboud Institute for Molecular Life Sciences, Radboud University Medical Center, Nijmegen, The Netherlands
${ }^{3}$ Department of Ophthalmology, University Hospital of Cologne, Cologne, Germany
${ }^{4}$ Department for Health and Evidence, Radboud University Medical Center, Nijmegen, The Netherlands
${ }^{5}$ Department of Human Genetics, Radboud University Medical Center, Nijmegen, The Netherlands
}

Correspondence: Anneke I. den Hollander, Radboud University Medical Center, Department of Ophthalmology, internal zip: 409, Philips van Leydenlaan 15, 6525 EX, Nijmegen, The Netherlands;

Anneke.denHollander@radboudumc $\mathrm{nl}$.

Submitted: April 7, 2015

Accepted: October 19, 2015

Citation: Paun CC, Ersoy L, Schick T, et al. Genetic variants and systemic complement activation levels are associated with serum lipoprotein levels in age-related macular degeneration. Invest Ophthalmol Vis Sci. 2015;56:7766-7773. DOI:10.1167/ iovs. $15-17035$
Purpose. Genetic variants in genes encoding components of lipid metabolism have been associated with AMD. The aims of this study were to evaluate the relation of these genetic variants with serum lipid levels in AMD in a large case-control cohort $(n=3070)$ and to test for correlations between lipids and complement activation.

Methods. Single nucleotide polymorphisms (SNPs) in eight lipid metabolism genes, previously described to be associated with AMD, were genotyped and tested for their association in our case-control cohort. Serum apolipoprotein B (ApoB), apolipoprotein AI (Apo-AI), cholesterol, triglycerides (TG), high-density lipoprotein-cholesterol (HDLC), and complement activation levels (C3d/C3) were measured and tested for association with AMD. Non-HDL cholesterol and LDL were inferred based on the measurements of the other lipids and lipoproteins. General linear models and $\chi^{2}$ tests were used to evaluate the relation of SNPs and lipids/ lipoproteins to the disease as well as their interrelations.

Results. Significant genotypic associations with AMD were observed for SNPs in CETP, APOE, and FADS1. The serum levels of Apo-AI and HDLC were significantly higher in patients compared with controls. Triglycerides (TG) levels were lower in AMD compared with controls. A cumulative effect was observed for APOE and CETP genotypes on HDLC and ApoAI levels. Complement activation levels correlated positively with HDLC and Apo-AI, and negatively with TG. Both the lipids/lipoproteins and the complement activation levels associate independently to AMD.

Conclusions. This study bridges the gap between genetic associations and physiological lipid levels in AMD. Additionally, the observed correlations between complement activation and lipid levels link two major systems that previously were always assessed independently.

Keywords: SNP, lipoprotein, AMD, complement system, lipids

\begin{abstract}
$A^{2}$ ge-related macular degeneration (AMD) is a multifactorial, $\mathbf{A}_{\text {progressive disease and a leading cause of blindness in the }}$ elderly population. ${ }^{1,2}$ The strong genetic underpinnings of AMD based on genome-wide association studies (GWAS) broadly point toward the involvement of three systems in the pathogenesis of AMD: the complement system, lipid metabolism, and the extracellular matrix. ${ }^{3}$ Investigating the pathways identified by genetic associations has proven to be a fruitful research strategy in the past. A higher rate of systemic complement activation levels was demonstrated in patients compared with controls, ${ }^{4,5}$ bringing systemic physiological consequences in line with the genetic associations. For the second major system involved in AMD, the lipid metabolism, such congruency is not immediately apparent.

Lipids, due to their insoluble nature, are transported through the circulation by lipoproteins. ${ }^{6}$ Two major lipoproteins of this process are low-density lipoprotein (LDL) and high-
\end{abstract}

density lipoprotein (HDL). ${ }^{7}$ Low-density lipoprotein is responsible for transporting cholesterol from the liver to the periphery, while HDL transports peripheral cholesterol back to the liver in a process called reverse cholesterol transport (RCT). ${ }^{8}$

In a recent GWAS on AMD, three genes involved in the lipoprotein transport system (CETP, APOE, and $L I P C$ ) reached genome-wide significance. ${ }^{3}$ In addition, two earlier association studies also reported associations for $L P L, F A D S 1$, and $A B C A 1{ }^{9,10}$ All of the proteins encoded by these genes, are either enzymes, coenzymes, or transporters within the lipid metabolism. Thus, ample genetic evidence exists for the involvement of lipid metabolism in the etiology of AMD.

In the pathology of AMD, aberrant lipid homeostasis has also been observed. Particularly, approximately $40 \%$ of drusen composition (one of the major hallmarks of AMD) is made up of esterified cholesterol, unesterified cholesterol, and phospha- 
tidylcholine. ${ }^{11}$ However, it has proven to be challenging to attribute risk scores for the development of AMD to systemic measurements of HDL or LDL. Studies directly investigating the levels of HDL/HDLC and LDL report conflicting results, with some presenting higher levels of HDL/HDLC in AMD patients compared with controls, ${ }^{12-18}$ whereas others describe the opposite. ${ }^{19-21}$ Yet other reports, including those that combined multiple of the previous studies, did not observe any significant differences between patients and controls. ${ }^{1,22-26}$

It is important to clarify the role of HDL and other lipids/ lipoproteins in AMD and their relation to the established AMDassociated lipid genes, as this may shed more light on the pathogenesis of AMD. Such insights potentially could lead to targeted and more efficient approaches toward treatment regimens for AMD. Therefore, the primary aim of this study was to investigate the relation of single nucleotide polymorphisms (SNPs) genotypes in the AMD-associated lipid genes and serum lipid levels in AMD in a large case-control cohort $(N$ $=3070$ ). The secondary aim was to determine if there is a correlation between the previously described complement activation $^{4}$ and lipid levels.

\section{MeTHODS}

\section{Study Population}

From the European Genetic Database (EUGENDA, in the public domain, www.eugenda.org), 3070 participants above the age of 50 years were included in the study. The study was performed in accordance with the tenets of the Declaration of Helsinki and the Medical Research Involving Human Subjects Act (WMO), and was approved by the local ethics committee of the University Hospitals in Cologne and Nijmegen. Written informed consent was obtained from all participants.

Age-related macular degeneration and control status were assigned by multimodal image grading that included stereo fundus photographs, fluorescein angiograms, and spectraldomain optical coherence tomograms. The grading was performed according to the standard protocol of the Cologne Image Reading Center (CIRCL) by certified graders (TR, LE). The classification of AMD was performed as described previously. ${ }^{27}$

Demographic data and nongenetic parameters including smoking status (current/past/never), regular alcohol intake (current/past/never), body mass index (BMI), exercise/physical activity (never, almost never, 1-2 times a week, 3 or more times a week), and daily fat consumption (more than $35 \mathrm{~g}$ oil per day: Yes/No) were obtained by standardized interviewerassisted questionnaires.

\section{Serum Measurements and Genetic Analysis}

Serum samples were used for the various lipid and systemic complement measurements. Serum was obtained by a standard coagulation/centrifugation protocol, after which the samples were stored at $-80^{\circ} \mathrm{C}$ within 1 hour after collection. Serum levels of apolipoprotein B (Apo-B), apolipoprotein AI (Apo-AI), total cholesterol, triglycerides (TG), and HDL-cholesterol (HDLC) were measured in all patients and controls using standard procedures by a clinical chemistry laboratory (Architect Analyzer; Abbott Diagnostics Hoofddorp, The Netherlands). Non-HDL cholesterol (NHDL) was calculated by subtracting HDLC from total cholesterol; and low-density lipoprotein (LDL) cholesterol was calculated using the Friedewald formula. ${ }^{28}$

Complement component $\mathrm{C} 3$ and the activation fragment C3d were measured in serum samples as previously de- scribed $^{29}$ and $\mathrm{C} 3 \mathrm{~d} / \mathrm{C} 3$ was calculated as a measure of complement activation.

Genomic DNA was extracted from peripheral blood samples using standard procedures. Eight SNPs in the LIPC, CETP, APOE, FADS1, LPL, and ABCA1 genes (see Supplementary Table S1) were genotyped using the KASPar SNP Genotyping System by LGC Genomics.

\section{Statistical Analysis}

All calculations were performed using SPSS software version 20.0 (IBM Software and Systems, Armonk, NY, USA). Associations between lipid levels, SNP genotypes and disease status were analyzed using general linear models with each lipid, in turn, set as the dependent variable. The first model was built to find the association between lipids and disease status, the model was corrected for all possible confounders (see Table 3). The second model was built to find the association between lipids and SNP's, again the model corrected for all possible confounders (see Table 4). In literature smoking status, alcohol intake, BMI, and dietary fat intake are reported to significantly influence lipid and lipoprotein levels, ${ }^{30-34}$ for this reason they were selected as correction factors for the models to eliminate any possible confounding. Additionally, age, sex, and exercise/ physical activity were significantly different between patients and controls, thus they were also added as correction factors.

In order to assess the cumulative effect of $C E T P$ and $A P O E$ SNPs on lipid/lipoprotein levels, a new variable was created that had all nine possible genotype combinations (see Table 4). The association with the lipid/lipoprotein levels was tested in a general linear model also corrected for age, sex, BMI, smoking status, alcohol intake, exercise/physical activity, daily fat consumption, and disease status. The significance threshold was corrected for multiple testing, $P$ values less than or equal to 0.006 ( $0.05 / 8$ associations per experiment) were considered statistically significant for the associations to AMD of both SNP genotypes and serum lipid levels. For the associations of the SNPs and serum lipid levels we have corrected for 12 associations ( 4 genetic variables against 3 lipid/lipoprotein levels). The $P$ values less than or equal to $0.004(0.05 / 12$ studied associations) were considered significant.

Associations between AMD phenotype and genotypes were evaluated using cross tabulation, $P$ values were calculated with Pearson $\chi^{2}$ and odds ratios were generated using logistic regression. Pearson correlations were used to investigate the relationship between lipids and complement activation levels.

All power calculations were performed using CaTS - Power Calculator v0.0.2 (Center for Statistical Genomics, University of Michigan, Ann Arbor, MI, USA) as previously described. ${ }^{35}$ For the calculation we assumed a multiplicative model, a disease prevalence of $10 \%$ and a significance level of $0.006(0.05 / 8$ SNPs). The disease allele frequency and genotype relative risk were extracted from the papers that first described the associations (Supplementary Table S1).

\section{Results}

Summary of the demographics for the subjects included in the present study are shown in Table 1.

Eight SNPs in genes of the lipid metabolism, previously shown to be associated with AMD, were selected from literature (see Supplementary Table S1). Out of the eight SNPs that were analyzed, genotypes in $C E T P(\mathrm{rs} 3764261 ; P=0.002)$, $A P O E$ (rs4420638; $P=0.005$ ), and FADS1 (rs174547; $P=$ 0.005), were significantly associated with AMD after correcting for multiple testing $(P<0.006)$. A summary of all associations and genotype frequencies are presented in Table 2. 
TABLE 1. Baseline Characteristics of Study Subjects

\begin{tabular}{lcc}
\hline & \multicolumn{2}{c}{ EUGENDA } \\
\cline { 2 - 3 } & AMD, & Control, \\
& $\boldsymbol{n}=\mathbf{1 4 9 1}$ & $\mathbf{n}=\mathbf{1 5 7 9}$ \\
\hline Female sex, \% & 60 & 58 \\
Mean age \pm SD, y & $74.7 \pm 8.4$ & $69 \pm 7.6$ \\
Age range, y & $50-101$ & $50-100$ \\
Mean BMI \pm SD & $25.82 \pm 3.8$ & $25.79 \pm 3.9$ \\
Smoking status, \% & & \\
Current & 10.2 & 8.5 \\
Past & 47.6 & 47.5 \\
Never & 42.2 & 44 \\
Regular alcohol intake, \% & & \\
Current & 57.6 & 56.4 \\
Past & 5 & 4.2 \\
Never & 37.4 & 39.4 \\
Exercise/physical activity, \% & & \\
Never & 24 & 6.8 \\
Almost never & 14.6 & 47 \\
1-2 times a week & 44.2 & 26.1 \\
3 or more times a week & 17.2 & 30.6 \\
Daily fat consumption, \% & & 69.4 \\
Yes & 28.8 & \\
No & 71.2 & \\
\hline
\end{tabular}

Significant differences between the two groups were only for age $(P$ $\left.=1.98 \times 10^{-114}\right), \operatorname{sex}(P=0.043)$, and exercise/physical activity $(P=9.9$ $\left.\times 10^{-25}\right)$.
Mean serum levels of Apo-B, Apo-AI, total cholesterol, HDLC, LDL, NHDL, and triglycerides of AMD patients compared to controls are presented in Table 3. After adjusting for age, sex, BMI, smoking status, alcohol intake, exercise/ physical activity, and daily fat consumption, AMD patients had significantly higher Apo-AI $(P=0.002)$ and HDLC levels $(P=$ $\left.4.4 \times 10^{-5}\right)$ compared with controls. In contrast, patients had significantly lower serum levels of TG $\left(P=1.9 \times 10^{-4}\right)$ compared with controls. Significant positive correlations were observed between Apo-AI and HDLC, and negative correlations between HDLC and TG (Supplementary Fig. S1). The association between Apo-AI and AMD was independent of HDLC and TG. Similarly, the association of TG with AMD was independent of HDLC and Apo-AI. When correcting for Apo-AI and TG, the association of HDLC with AMD was negated, which is in line with the correlation of HDLC with Apo-AI and TG levels. No significant associations were found for any of the other measurements

Stratifying for the different AMD stages revealed significant associations only with the intermediate AMD stage. The observed effect directions were similar to the comparison of all AMD stages versus controls, with only Apo-AI, HDLC, and TG being significantly associated with intermediate AMD after correction for multiple testing (Supplementary Table S2).

The SNPs that were significantly associated with AMD in the EUGENDA cohort, were analyzed for association with the lipid/ lipoprotein levels that significantly differed between patients and controls. Only APOE (rs4420638) and CETP (rs3764261) genotypes displayed significant associations with Apo-AI and HDLC serum levels. APOE (rs4420638) genotypes were moderately associated with TG levels $(P=0.026)$, however the association did not remain significant after correcting for multiple testing. A summary of the results is presented in Table 4.

Because both CETP and APOE SNP genotypes were significantly associated with Apo-AI and HDLC serum levels,

TABLE 2. Association of Genotypes in Lipid Metabolism Genes With AMD

\begin{tabular}{|c|c|c|c|c|c|c|c|}
\hline \multirow[b]{2}{*}{ SNP } & \multirow[b]{2}{*}{ Gene } & \multirow[b]{2}{*}{$P^{*}$} & \multirow[b]{2}{*}{ Genotype } & \multicolumn{2}{|c|}{ Status, \% } & \multirow[b]{2}{*}{ OR } & \multirow[b]{2}{*}{$95 \% \mathrm{CI}$} \\
\hline & & & & Control & AMD & & \\
\hline \multirow[t]{3}{*}{ rs493258 } & LIPC & 0.388 & TT & 21.1 & 19.6 & & \\
\hline & & & CT & 49.0 & 48.6 & 1.07 & $0.89-1.27$ \\
\hline & & & CC & 29.9 & 31.8 & 1.14 & $0.94-1.38$ \\
\hline \multirow[t]{3}{*}{ rs10468017 } & LIPC & 0.161 & TT & 8.2 & 7.7 & & \\
\hline & & & CT & 41.9 & 39.1 & 0.99 & $0.76-1.28$ \\
\hline & & & $\mathrm{CC}$ & 49.9 & 53.2 & 1.13 & $0.88-1.46$ \\
\hline \multirow[t]{3}{*}{ rs3764261 } & CETP & 0.002 & GG & 47.8 & 42.3 & & \\
\hline & & & GT & 41.2 & 44.2 & 1.21 & $1.05-1.40$ \\
\hline & & & TT & 10.9 & 13.5 & 1.40 & $1.12-1.74$ \\
\hline \multirow[t]{3}{*}{ rs2075650 } & $A P O E$ & 0.043 & GG & 1.6 & 1.4 & & \\
\hline & & & GA & 23.5 & 20.1 & 0.96 & $0.54-1.69$ \\
\hline & & & AA & 74.9 & 78.5 & 1.18 & $0.68-2.05$ \\
\hline \multirow[t]{3}{*}{ rs4420638 } & $A P O E$ & 0.005 & GG & 3.0 & 1.9 & & \\
\hline & & & GA & 28.6 & 25.3 & 1.41 & $0.89-2.21$ \\
\hline & & & AA & 68.3 & 72.8 & 1.70 & $1.09-2.65$ \\
\hline \multirow{3}{*}{ rs12678919 } & $L P L$ & 0.579 & $\mathrm{AA}$ & 80.7 & 79.3 & & \\
\hline & & & $\mathrm{AG}$ & 18.1 & 19.4 & 1.10 & $0.92-1.30$ \\
\hline & & & GG & 1.3 & 1.2 & 0.99 & $0.54-1.81$ \\
\hline \multirow[t]{3}{*}{ rs174547 } & FADS1 & 0.005 & $\mathrm{CC}$ & 11.8 & 8.5 & & \\
\hline & & & CT & 43.1 & 43.8 & 1.41 & $1.12-1.79$ \\
\hline & & & TT & 45.1 & 47.7 & 1.47 & $1.16-1.86$ \\
\hline \multirow[t]{3}{*}{ rs3758294 } & $A B C A 1$ & 0.982 & TT & 63.1 & 62.9 & & \\
\hline & & & TC & 32.6 & 32.8 & 1.03 & $0.73-1.45$ \\
\hline & & & $\mathrm{CC}$ & 4.3 & 4.2 & 1.02 & $0.73-1.42$ \\
\hline
\end{tabular}

* After correction for multiple testing significance is reached at $P \leq 0.006$. 
TABLE 3. Association of Mean Serum Lipid/Lipoprotein Levels With AMD

\begin{tabular}{llrrc}
\hline \multicolumn{1}{c}{ Lipid } & Status & Mean & \multicolumn{1}{c}{ SE } & $\boldsymbol{P}$ \\
\hline Apo-B, mg/L & Control & 954.98 & 9.50 & 0.788 \\
& AMD & 957.44 & 9.41 & \\
Apo-AI, mg/L & Control & 1615.76 & 11.43 & 0.002 \\
Total cholesterol, mM & AMD & 1649.35 & 11.32 & \\
& Control & 5.60 & 0.05 & 0.155 \\
HDLC, mM & AMD & 5.66 & 0.05 & \\
LDL, mM & Control & 1.40 & 0.01 & $4.6 \times 10^{-5}$ \\
& AMD & 1.45 & 0.01 & \\
NHDL, mM & Control & 3.83 & 0.04 & 0.739 \\
TG, mM & AMD & 3.85 & 0.04 & \\
& Control & 4.20 & 0.05 & 0.819 \\
& AMD & 4.21 & 0.04 & \\
& Control & 1.76 & 0.03 & $1.9 \times 10^{-4}$ \\
& AMD & 1.65 & 0.03 & \\
& & & & \\
& & &
\end{tabular}

General linear models were built with each lipid/lipoprotein as the dependent variable. The models tested for the association to disease status and were all corrected for age, sex, BMI, smoking status, alcohol intake, exercise/physical activity and daily fat consumption. Threshold for statistical significance $P<0.006$.

the cumulative effect of carrying multiple risk genotypes on the lipid levels was investigated. Mean levels for each genotype combination of CETP and $A P O E$ are displayed in Table 4 and visualized in Figure 1. In both cases, carriers of double high-risk genotypes for $C E T P$ and $A P O E$ showed significantly elevated levels of Apo-AI and HDLC compared with low-risk genotype carriers.

To exclude the possibility that the associations of HDLC and Apo-AI with AMD were mainly a consequence of the underlying genetic associations of $C E T P$ and $A P O E$ that drive HDLC and Apo-AI levels, all lipid analyses were corrected for CETP and APOE genotypes and all the other genotyped SNPs. After doing so, HDLC and Apo-AI remained significantly associated with AMD, independent of the genotypes $(P=1.4$ $\times 10^{-4}$ and 0.003 , respectively)

Finally, because serum complement activation levels were previously shown to be associated to AMD,${ }^{4}$ we tested whether a relation exists between lipid levels and complement activation levels $(\mathrm{C} 3 \mathrm{~d} / \mathrm{C} 3$ ratio). This analysis revealed significant positive correlations between Apo-AI, HDLC, and complement activation and a significant negative correlation for TG (Fig. 2). All $P$ values were less than $1.9 \times 10^{-9}$. A general linear model corrected for disease status and other variables confirmed the association of $\mathrm{C} 3 \mathrm{~d} / \mathrm{C} 3$ to lipids/lipoproteins $(P=$ $\left.<1.9 \times 10^{-9}\right)$ and revealed that the association to disease status is independent of lipid levels $\left(P=9 \times 10^{-6}\right)$.

\section{Discussion}

The genetic analyses from the present case-control study confirm previously described associations for CETP (rs3764261), APOE (rs4420638), and FADS1 (rs174547) with AMD. However, no associations were observed for $A P O E$ (rs2075650), LIPC (rs493258 and rs10468017), LPL (rs12678919), and $A B C A 1$ (rs3758294). The SNPs were selected from recent large GWAS $^{3,9,10,36,37}$ (see Supplementary Table S1). For the SNPs in $A B C A 1$ (rs1883025) and LIPC (rs493258 and rs10468017) our study was underpowered with $52 \%, 59 \%$, and $53 \%$ chance of detection, respectively. Therefore, we cannot exclude the possibility that these SNPs may be associated to AMD in a larger cohort. On the other hand, for $L P L$ (rs12678919) this study had

TABLE 4. Association of AMD SNPs With Serum Lipid/Lipoprotein Levels

\begin{tabular}{|c|c|c|c|c|c|c|c|}
\hline \multirow[b]{3}{*}{ SNP } & \multirow[b]{3}{*}{ Genotype } & \multicolumn{6}{|c|}{ Lipid/Lipoprotein } \\
\hline & & \multicolumn{2}{|c|}{ Apo-AI, mg/L } & \multicolumn{2}{|c|}{ HDLC, mM } & \multicolumn{2}{|c|}{ TG, $\mathbf{m M}$} \\
\hline & & Mean & SE & Mean & SE & Mean & SE \\
\hline \multirow[t]{4}{*}{ CETP rs3764261 } & GG & 1601.6 & 12.1 & 1.37 & 0.01 & 1.71 & 0.03 \\
\hline & GT & 1645.9 & 12.0 & 1.46 & 0.01 & 1.71 & 0.03 \\
\hline & $\mathrm{TT}^{*}$ & 1704.6 & 17.8 & 1.54 & 0.02 & 1.73 & 0.05 \\
\hline & Sig. & \multicolumn{2}{|c|}{$2.8 \times 10^{-9}$} & \multicolumn{2}{|c|}{$6.50 \times 10^{-20}$} & \multicolumn{2}{|c|}{0.950} \\
\hline \multirow[t]{4}{*}{ APOE rs4420638 } & GG & 1555.7 & 35.9 & 1,32 & 0.04 & 1.95 & 0.09 \\
\hline & GA & 1612.6 & 13.8 & 1.42 & 0.02 & 1.73 & 0.04 \\
\hline & $\mathrm{AA}^{*}$ & 1643.6 & 11.0 & 1.44 & 0.01 & 1.71 & 0.03 \\
\hline & Sig. & \multicolumn{2}{|c|}{0.003} & \multicolumn{2}{|c|}{0.012} & \multicolumn{2}{|c|}{0.026} \\
\hline \multirow[t]{4}{*}{ FADS1 rs174547 } & $\mathrm{CC}$ & 1620.4 & 18.6 & 1.44 & 0.02 & 1.75 & 0.05 \\
\hline & $\mathrm{TC}$ & 1632.8 & 12.1 & 1.43 & 0.01 & 1.74 & 0.03 \\
\hline & $\mathrm{TT}^{\mathrm{a}}$ & 1638.5 & 12.0 & 1.43 & 0.01 & 1.69 & 0.03 \\
\hline & Sig. & \multicolumn{2}{|c|}{0.595} & \multicolumn{2}{|c|}{0.832} & \multicolumn{2}{|c|}{0.137} \\
\hline \multirow[t]{10}{*}{ CETP/APOE } & GG/GG & 1531.3 & 48.8 & 1.26 & 0.06 & 2.1 & 0.13 \\
\hline & GG/GA & 1561.4 & 18.0 & 1.33 & 0.02 & 1.7 & 0.05 \\
\hline & GG/AA & 1619.0 & 13.1 & 1.39 & 0.02 & 1.7 & 0.04 \\
\hline & GT/GG & 1590.9 & 54.6 & 1.41 & 0.06 & 1.8 & 0.14 \\
\hline & GT/GA & 1651.7 & 18.2 & 1.48 & 0.02 & 1.7 & 0.05 \\
\hline & GT/AA & 1644.2 & 13.0 & 1.45 & 0.02 & 1.7 & 0.03 \\
\hline & $\mathrm{TT} / \mathrm{GG}$ & 1530.6 & 123.3 & 1.29 & 0.14 & 2.2 & 0.36 \\
\hline & TT/GA & 1660.3 & 29.7 & 1.51 & 0.03 & 1.8 & 0.08 \\
\hline & TT/AA & 1725.3 & 20.7 & 1.56 & 0.02 & 1.7 & 0.05 \\
\hline & Sig. & \multicolumn{2}{|c|}{$1.3 \times 10^{-9}$} & \multicolumn{2}{|c|}{$4.4 \times 10^{-19}$} & \multicolumn{2}{|c|}{0.118} \\
\hline
\end{tabular}

The model was corrected for age, sex, BMI, smoking status, alcohol intake, exercise/physical activity, daily fat consumption, and disease status. Threshold for statistical significance $P<0.004$. Sig., significance.

${ }^{*}$ Risk allele for AMD in our cohort. 
A

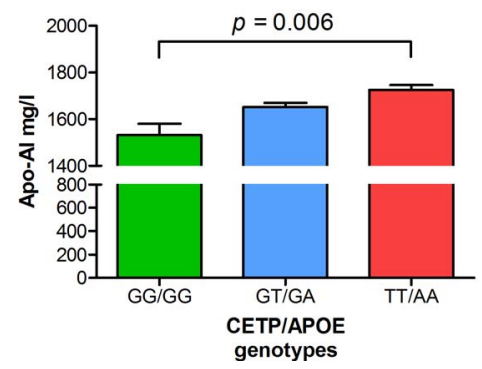

B

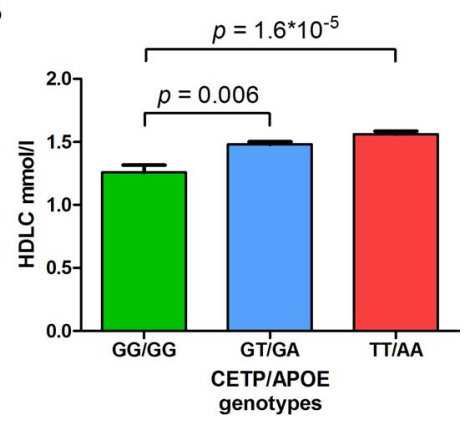

FIGURE 1. Mean lipid levels with standard error bars for CETP/APOE homozygous high-risk genotypes, heterozygous and homozygous low-risk genotypes. All $P$ values are Bonferroni-corrected. (A) Levels for Apo-AI; (B) levels for HDLC.

$77 \%$ detection power, suggesting that not all genetic associations may be reliably replicated between different populations.

In this study, we observed significant differences in the serum levels of Apo-AI, HDLC, and TG of AMD patients compared with controls. Triglycerides were significantly lower, while Apo-AI and HDLC were significantly higher in patients compared with controls. No statistically significant associations with AMD were detected for any of the other measured lipids/ lipoproteins.

In the literature, there are inconsistent associations of AMD with serum lipid levels. Comparing the mean lipid/lipoprotein levels observed in the present study with values previously reported (Supplementary Table S3), is challenging since the measurements were performed differently across the various reports, different correction factors were applied, and different populations were studied. All of these factors can influence the mean levels, making it difficult to pinpoint the cause of the different study outcomes. However, if we compare the main effects, our findings of high HDLC levels in patients compared with controls are consistent with several previous studies. ${ }^{12-18}$ The positive association of HDLC with only the intermediate AMD stage confirms the finding reported by CougnardGrégoire et al. ${ }^{12}$ On the other hand, other publications have reported inverse or no association between HDLC and AMD. ${ }^{19-23,25,26}$ When results were pooled in a meta-analysis, no associations have been detected. ${ }^{1,24,38}$ To our knowledge, for Apo-AI this is the first large study to report a positive association with AMD, and for TG other studies reported opposite or no associations with AMD. ${ }^{12,20,26}$ The reasons for these inconsistencies are not fully understood, however in a recent publication high levels of HDLC were associated with risk for AMD only after a stringent multivariate correction. ${ }^{12}$ Because our study, and others, ${ }^{39-41}$ show a clear effect of genotype on lipid levels, correcting for these genotypes may improve the insight into the associations of lipid levels with AMD and the direction of their effect. This is especially important because our study, although appropriately powered, had failed to detect associations with LPL (rs12678919), suggesting that population- or cohort-specific genetic substructures may account partly for the observed inconsistencies. Another reason could be related to sample size, which in some studies might not be large enough to allow for the necessary adjustments and still have sufficient power to detect significant associations. In our cohort, higher levels of Apo-AI and HDLC were associated with risk genotypes in CETP (rs3764261; TT) and $A P O E$ (rs4420638; AA). A cumulative effect was observed for these two SNPs, with a risk-allele dose dependent increase in both HDLC and Apo-AI serum levels (Fig. 1). The CETP and $A P O E$ loci have previously been linked to lipid metabolism in cardiovascular studies. ${ }^{40}$ In the context of AMD, few studies have looked into the relation of AMD lipid SNPs and serum lipid levels. Our results for CETP were consistent with a recent report from the Alienor study. ${ }^{12}$ Another study observed that in individuals carrying the LPL (rs12678919) GG genotype, TG levels were significantly lower and HDLC levels were significantly higher. ${ }^{42}$ Moreover, one study reported that the LIPC (rs10468017) T allele was associated with higher levels of HDLC. ${ }^{43}$ Our study does not describe an association of $L P L$ and LIPC genotypes with lipid levels, because no significant difference between patients and controls was observed.

CETP encodes for cholesterol ester transfer protein (CETP), which promotes the transfer of excess cholesterol ester (CE) to the liver through the RCT pathway. ${ }^{44}$ Several studies have shown that lower CETP activity leads to higher HDLC levels. ${ }^{41,45,46}$ APOE encodes for apolipoprotein E (ApoE), which plays a major role in the metabolism of cholesterol and TG by mediating the clearance of chylomicrons and very low-density-lipoprotein (VLDL) from the bloodstream. ${ }^{47,48}$ ApoE has been described to have a direct relation with CETP by enhancing the CE and TG transfer between VLDL and HDL in a CETP-dependent manner. ${ }^{49}$ Despite the direct impact of APOE and CETP on HDLC metabolism, understanding how the
A

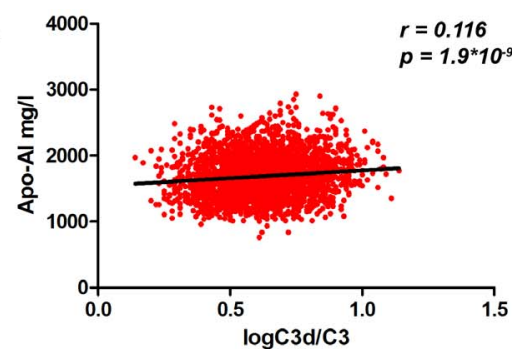

B

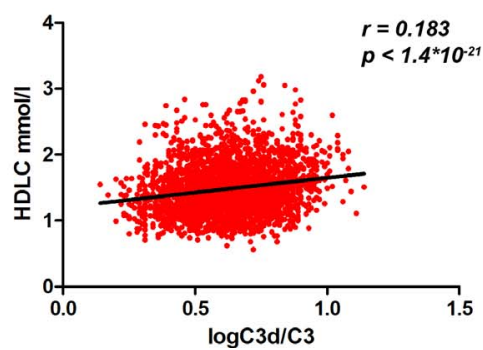

C

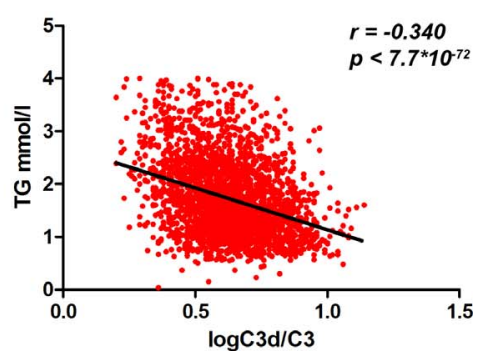

FIGURE 2. Scatterplots showing the correlations of lipid levels with complement activation levels represented by the log transformed ratio of C3d/ C3. Direction of the correlations is indicated by the black regression line. (A) Positive correlation of Apo-AI and logC3d/C3; (B) positive correlation of HDLC with $\log \mathrm{C} 3 \mathrm{~d} / \mathrm{C} 3$; (C) negative correlation of TG and $\log \mathrm{C} 3 \mathrm{~d} / \mathrm{C} 3$. 
risk genotypes of the studied SNPs could have the cumulative HDLC raising effect is not directly obvious, mainly because both rs3764261 and rs4420638 are located in intergenic regions. One possibility may be a consequence of an effect on CETP expression levels that was reported for $\mathrm{rs} 3764261 .^{50}$

Traditionally, rs4420638 is reported as an $A P O E$ SNP, because it is considered a proxy for rs429358, one of the two coding variants that determine the APOE isoforms $(\varepsilon 2, \varepsilon 3$, and $\varepsilon 4$ ) reported to attenuate binding affinity to the (LDL)receptor, ${ }^{51}$ and thus affect the entire cholesterol metabolism. However, the $r^{2}$ value for the linkage disequilibrium of these two SNPs is $0.63,{ }^{3}$ indicating that there is not a complete coinheritance. Also its genomic position is closer to the APOC1 gene, a potent inhibitor of CETP activity, ${ }^{52}$ thus we cannot exclude the possibility of rs4420638 for being a proxy for a regulatory variant of $A P O C 1$ instead.

Understanding the local involvement of lipid and lipoprotein systems at AMD disease sites in the eye is made difficult by the lack of information regarding eye specific function of these molecules. Nevertheless, if we focus on HDLC metabolism, clues can be found. First, key components of the RCT pathway for which the main player is HDLC, ${ }^{53}$ are expressed in the retina. ${ }^{54-56}$ In addition, during the normal aging process, an accumulation of Apo-B of unusual composition takes place in the Bruch's membrane, forming a precursor of basal linear deposit, called the "lipid wall." 57 Moreover, the macromolecular conductivity of the Bruch's membrane reduces 10-fold between the first and ninth decades of life, which is significant because lipoproteins need to cross the Bruch's membrane in order to mediate lipid efflux from the RPE. ${ }^{58,59}$ Furthermore, in vitro, HDL has been observed to mediate efflux of photoreceptor outer segment lipids from the basal surfaces of RPE cells. ${ }^{60}$ Finally, a retention of cholesterol in drusen, the major lesions of AMD, has been reported. ${ }^{61}$

Besides the involvement of HDL in lipid and lipoprotein transport, this system has recently been implicated in immune function. ${ }^{62}$ Recent proteomic analyses revealed several types of HDL particles containing complement system components $\mathrm{C} 4 \mathrm{a}, \mathrm{C} 4 \mathrm{~b}, \mathrm{C} 9$, and vitronectin ${ }^{63,64}$ in healthy subjects, and $\mathrm{C} 3$ in patients with coronary artery disease. ${ }^{63}$ In our study, we offer support for this emerging concept by demonstrating a significant correlation between HDLC and complement system activation, although it remains to be determined whether the effect is direct or indirect.

One possible limitation of our study may be that the lipid/ lipoprotein levels were not overnight fasted blood measurements, which could induce possible artifacts for certain lipids like the TG. However, the fact that HDLC and Apo-AI levels are not severely affected by food intake, ${ }^{65}$ and the great number of participants in this study, negate this potential drawback.

In our study, the genetic and environmental factors explain $31 \%, 84 \%$, and $27 \%$ of the Apo-AI, HDLC, and TG variation, respectively. Our findings indicate that other factors must be associated with them, which might relate to the AMD disease pathogenesis.

In conclusion, the results of our study indicate that patients with high risk CETP/APOE genotypes and high HDLC levels have higher risk of developing AMD, suggesting that they could potentially benefit from HDLC lowering regimens. Further studies are needed to investigate the role of HDL subfractions and the observed correlation of HDLC with complement activation in the disease pathogenesis of AMD.

\section{Acknowledgments}

The authors thank Frederieke Schoenmaker-Koller, Bjorn Bakker, and Bert Janssen for excellent technical support.
Supported by the Foundation Fighting Blindness Center Grant to the Radboud University Medical Center (Grant C-GE-0811-0548RAD04; Columbia, MD, USA) and the Netherlands Organization for Scientific Research (Vidi Innovational Research Award 016.096.309 to AIdH; Den Haag, Zuid-Holland, The Netherlands).

Disclosure: C.C. Paun, None; L. Ersoy, None; T. Schick, None; J.M.M. Groenewoud, None; Y.T. Lechanteur, None; S. Fauser, None; C.B. Hoyng, None; E.K. de Jong, None; A.I. den Hollander, None

\section{References}

1. Smith W, Assink J, Klein R, et al. Risk factors for age-related macular degeneration: pooled findings from three continents. Ophthalmology. 2001;108:697-704.

2. Klein R, Klein BE, Linton KL. Prevalence of age-related maculopathy. The Beaver Dam Eye Study. Ophthalmology. 1992;99:933-943.

3. Fritsche LG, Chen W, Schu M, et al. Seven new loci associated with age-related macular degeneration. Nat Genet. 2013;45: 433-439, 439e431-432.

4. Ristau T, Paun C, Ersoy L, et al. Impact of the common genetic associations of age-related macular degeneration upon systemic complement component C3d levels. PLoS One. 2014;9: e93459.

5. Scholl HP, Charbel Issa P, Walier M, et al. Systemic complement activation in age-related macular degeneration. PLoS One. 2008;3:e2593.

6. Lusis AJ, Pajukanta P. A treasure trove for lipoprotein biology. Nat Genet. 2008;40:129-130.

7. Mahley RW, Innerarity TL, Rall SC Jr, Weisgraber KH. Plasma lipoproteins: apolipoprotein structure and function. J Lipid Res. 1984;25:1277-1294.

8. Birner-Gruenberger R, Schittmayer M, Holzer M, Marsche G. Understanding high-density lipoprotein function in disease: recent advances in proteomics unravel the complexity of its composition and biology. Prog Lipid Res. 2014;56:36-46.

9. Neale BM, Fagerness J, Reynolds R, et al. Genome-wide association study of advanced age-related macular degeneration identifies a role of the hepatic lipase gene (LIPC). Proc Natl Acad Sci U S A. 2010;107:7395-7400.

10. Chen W, Stambolian D, Edwards AO, et al. Genetic variants near TIMP3 and high-density lipoprotein-associated loci influence susceptibility to age-related macular degeneration. Proc Natl Acad Sci US A. 2010;107:7401-7406.

11. Wang L, Clark ME, Crossman DK, et al. Abundant lipid and protein components of drusen. PLoS One. 2010;5:e10329.

12. Cougnard-Gregoire A, Delyfer MN, Korobelnik JF, et al. Elevated high-density lipoprotein cholesterol and age-related macular degeneration: the Alienor study. PLoS One. 2014;9: e90973.

13. Butt AL, Lee ET, Klein R, et al. Prevalence and risks factors of age-related macular degeneration in Oklahoma Indians: the Vision Keepers Study. Ophthalmology. 2011;118:1380-1385.

14. Delcourt C, Michel F, Colvez A, et al. Associations of cardiovascular disease and its risk factors with age-related macular degeneration: the POLA study. Ophthalmic Epidemiol. 2001;8:237-249.

15. Hyman L, Schachat AP, He Q, Leske MC. Hypertension, cardiovascular disease, and age-related macular degeneration. Age-Related Macular Degeneration Risk Factors Study Group. Arch Ophthalmol. 2000;118:351-358.

16. Klein R, Klein BE, Franke T. The relationship of cardiovascular disease and its risk factors to age-related maculopathy. The Beaver Dam Eye Study. Ophthalmology. 1993;100:406-414.

17. Klein R, Klein BE, Tomany SC, Cruickshanks KJ. The association of cardiovascular disease with the long-term 
incidence of age-related maculopathy: the Beaver Dam Eye Study. Ophthalmology. 2003;110:1273-1280.

18. van Leeuwen R, Klaver CC, Vingerling JR, et al. Cholesterol and age-related macular degeneration: is there a link? $A m \mathrm{~J}$ Ophthalmol. 2004;137:750-752.

19. Klein R, Cruickshanks KJ, Nash SD, et al. The prevalence of age-related macular degeneration and associated risk factors. Arch Opbthalmol. 2010;128:750-758.

20. Nowak M, Swietochowska E, Marek B, et al. Changes in lipid metabolism in women with age-related macular degeneration. Clin Exp Med. 2005;4:183-187.

21. Tan JS, Mitchell P, Smith W, Wang JJ. Cardiovascular risk factors and the long-term incidence of age-related macular degeneration: the Blue Mountains Eye Study. Ophthalmology. 2007; 114:1143-1150.

22. Fauser S, Smailhodzic D, Caramoy A, et al. Evaluation of serum lipid concentrations and genetic variants at high-density lipoprotein metabolism loci and TIMP3 in age-related macular degeneration. Invest Ophthalmol Vis Sci. 2011;52:5525-5528.

23. Cackett P, Wong TY, Aung T, et al. Smoking, cardiovascular risk factors, and age-related macular degeneration in Asians: the Singapore Malay Eye Study. Am J Ophthalmol. 2008;146:960967, e961.

24. Klein R, Myers CE, Buitendijk GH, et al. Lipids, lipid genes, and incident age-related macular degeneration: the three continent age-related macular degeneration consortium. Am J Ophthalmol. 2014; 158:513-524, e513.

25. Ulas F, Balbaba M, Ozmen S, Celebi S, Dogan U. Association of dehydroepiandrosterone sulfate, serum lipids, C-reactive protein and body mass index with age-related macular degeneration. Int Ophthalmol. 2013;33:485-491.

26. Davari MH, Gheitasi H, Yaghobi G, Heydari B. Correlation between serum lipids and age-related macular degeneration: a case-control study. J Res Health Sci. 2012;13:98-101.

27. Ristau T, Ersoy L, Lechanteur Y, et al. Allergy is a protective factor against age-related macular degeneration. Invest Ophthalmol Vis Sci. 2014;55:210-214.

28. Friedewald WT, Levy RI, Fredrickson DS. Estimation of the concentration of low-density lipoprotein cholesterol in plasma, without use of the preparative ultracentrifuge. Clin Chem. 1972;18:499-502

29. Smailhodzic D, Klaver CC, Klevering BJ, et al. Risk alleles in CFH and ARMS2 are independently associated with systemic complement activation in age-related macular degeneration. Ophthalmology. 2012;119:339-346.

30. Chrysohoou C, Panagiotakos DB, Pitsavos C, et al. Effects of chronic alcohol consumption on lipid levels, inflammatory and haemostatic factors in the general population: the 'ATTICA' Study. Euro J Cardiovasc Prev Rehab. 2003;10: 355-361.

31. Craig WY, Palomaki GE, Haddow JE. Cigarette smoking and serum lipid and lipoprotein concentrations: an analysis of published data. BMJ. 1989;298:784-788.

32. Shamai L, Lurix E, Shen M, et al. Association of body mass index and lipid profiles: evaluation of a broad spectrum of body mass index patients including the morbidly obese. Obes Surg. 2011;21:42-47.

33. Toeller M, Buyken AE, Heitkamp G, Scherbaum WA, Krans HM, Fuller JH. Associations of fat and cholesterol intake with serum lipid levels and cardiovascular disease: the EURODIAB IDDM Complications Study. Exp Clin Endocrinol Diabetes.1999;107: 512-521

34. Bel-Serrat S, Mouratidou T, Huybrechts I, et al. Associations between macronutrient intake and serum lipid profile depend on body fat in European adolescents: the Healthy Lifestyle in Europe by Nutrition in Adolescence (HELENA) study. $\mathrm{Br} \mathrm{J}$ Nutr. 2014;112:2049-2059.
35. Skol AD, Scott LJ, Abecasis GR, Boehnke M. Joint analysis is more efficient than replication-based analysis for two-stage genome-wide association studies. Nat Genet. 2006;38:209213.

36. Deelen J, Beekman M, Uh HW, et al. Genome-wide association study identifies a single major locus contributing to survival into old age; the APOE locus revisited. Aging Cell. 2011;10: 686-698.

37. Holliday EG, Smith AV, Cornes BK, et al. Insights into the genetic architecture of early stage age-related macular degeneration: a genome-wide association study meta-analysis. PLoS One. 2013;8:e53830.

38. Chakravarthy U, Wong TY, Fletcher A, et al. Clinical risk factors for age-related macular degeneration: a systematic review and meta-analysis. BMC Ophthalmol. 2010;10:31.

39. Aledo R, Padro T, Mata P, Alonso R, Badimon L. rs11613352 polymorphism (TT genotype) associates with a decrease of triglycerides and an increase of HDL in familial hypercholesterolemia patients. Rev Esp Cardiol. 2014;68:305-309.

40. Global Lipids Genetics Consortium, Willer CJ, Schmidt EM, et al. Discovery and refinement of loci associated with lipid levels. Nat Genet. 2013;45:1274-1283.

41. Inazu A, Brown ML, Hesler CB, et al. Increased high-density lipoprotein levels caused by a common cholesteryl-ester transfer protein gene mutation. $N$ Engl J Med. 1990;323: 1234-1238.

42. Merle BM, Maubaret C, Korobelnik JF, et al. Association of HDL-related loci with age-related macular degeneration and plasma lutein and zeaxanthin: the Alienor study. PLoS One. 2013;8:e79848.

43. Reynolds R, Rosner B, Seddon JM. Serum lipid biomarkers and hepatic lipase gene associations with age-related macular degeneration. Ophthalmology. 2010;117:1989-1995.

44. de Grooth GJ, Klerkx AH, Stroes ES, Stalenhoef AF, Kastelein JJ, Kuivenhoven JA. A review of CETP and its relation to atherosclerosis. J Lipid Res. 2004;45:1967-1974.

45. Brown ML, Inazu A, Hesler CB, et al. Molecular basis of lipid transfer protein deficiency in a family with increased highdensity lipoproteins. Nature. 1989;342:448-451.

46. Inazu A, Jiang XC, Haraki T, et al. Genetic cholesteryl ester transfer protein deficiency caused by two prevalent mutations as a major determinant of increased levels of high density lipoprotein cholesterol. J Clin Invest. 1994;94:18721882.

47. Sun L, Hu C, Zheng C, et al. Gene-gene interaction between CETP and APOE polymorphisms confers higher risk for hypertriglyceridemia in oldest-old Chinese women. Exp Gerontol. 2014;55:129-133.

48. Sorli JV, Corella D, Frances F, et al. The effect of the APOE polymorphism on HDL-C concentrations depends on the cholesterol ester transfer protein gene variation in a Southern European population. Clin Chim Acta. 2006;366:196-203.

49. Kinoshita M, Arai H, Fukasawa M, et al. Apolipoprotein E enhances lipid exchange between lipoproteins mediated by cholesteryl ester transfer protein. J Lipid Res. 1993;34:261268.

50. Papp AC, Pinsonneault JK, Wang D, et al. Cholesteryl ester transfer protein (CETP) polymorphisms affect mRNA splicing, HDL levels, and sex-dependent cardiovascular risk. PLoS One. 2012;7:e31930.

51. McKay GJ, Patterson CC, Chakravarthy U, et al. Evidence of association of APOE with age-related macular degeneration: a pooled analysis of 15 studies. Hum Mutat. 2011;32:14071416.

52. Gautier T, Masson D, de Barros JP, et al. Human apolipoprotein C-I accounts for the ability of plasma high density lipoproteins to inhibit the cholesteryl ester transfer protein activity. J Biol Chem. 2000;275:37504-37509. 
53. Kontush A. HDL-mediated mechanisms of protection in cardiovascular disease. Cardiovasc Res. 2014;103:341-349.

54. Ishida BY, Bailey KR, Duncan KG, et al. Regulated expression of apolipoprotein $\mathrm{E}$ by human retinal pigment epithelial cells. J Lipid Res. 2004; 45:263-271.

55. Duncan KG, Bailey KR, Kane JP, Schwartz DM. Human retinal pigment epithelial cells express scavenger receptors BI and BII. Biochem Biophys Res Commun. 2002;292:1017-1022.

56. Duncan KG, Hosseini K, Bailey KR, et al. Expression of reverse cholesterol transport proteins ATP-binding cassette A1 (ABCA1) and scavenger receptor BI (SR-BI) in the retina and retinal pigment epithelium. Br J Ophthalmol. 2009;93:11161120.

57. Curcio CA, Johnson M, Rudolf M, Huang JD. The oil spill in ageing Bruch membrane. Br J Ophthalmol. 2011;95:1638-1645.

58. Moore DJ, Clover GM. The effect of age on the macromolecular permeability of human Bruch's membrane. Invest Ophthalmol Vis Sci. 2001;42:2970-2975.

59. Kishan AU, Modjtahedi BS, Martins EN, Modjtahedi SP, Morse LS. Lipids and age-related macular degeneration. Surv Ophthalmol. 2011;56:195-213.
60. Ishida BY, Duncan KG, Bailey KR, Kane JP, Schwartz DM. High density lipoprotein mediated lipid efflux from retinal pigment epithelial cells in culture. Br J Ophthalmol. 2006;90:616-620.

61. Pikuleva IA, Curcio CA. Cholesterol in the retina: the best is yet to come. Prog Retin Eye Res. 2014;41:64-89.

62. Norata GD, Pirillo A, Ammirati E, Catapano AL. Emerging role of high density lipoproteins as a player in the immune system. Atherosclerosis. 2012;220:11-21.

63. Vaisar T, Pennathur S, Green PS, et al. Shotgun proteomics implicates protease inhibition and complement activation in the antiinflammatory properties of HDL. J Clin Invest. 2007; 117:746-756.

64. Gordon SM, Deng J, Lu LJ, Davidson WS. Proteomic characterization of human plasma high density lipoprotein fractionated by gel filtration chromatography. J Proteome Res. 2010;9:5239-5249.

65. Langsted A, Freiberg JJ, Nordestgaard BG. Fasting and nonfasting lipid levels: influence of normal food intake on lipids, lipoproteins, apolipoproteins, and cardiovascular risk prediction. Circulation. 2008;118:2047-2056. 